\title{
Physics Potential of Solar Neutrino Experiments
}

\author{
A.B. Balantekin ${ }^{\mathrm{a} *}$, H. Yüksel ${ }^{\mathrm{a}}$

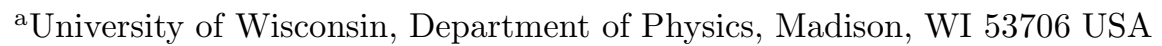

We discuss the physics potential of the solar neutrino experiments i) To explore the parameter space of neutrino mass and mixings; ii) To probe the physics of the Sun; iii) To explore nuclear physics of the neutrino-target interactions. Examples are given for these three classes.

Recently announced results from the Sudbury Neutrino Observatory (SNO) 1 and KamLAND [2] experiments indicate that neutrino physics is moving from the discovery stage to the precision measurements stage. (For recent reviews see e. g. 34). A combined analysis of the data from these experiments as well as data from other solar neutrino experiments (Super-Kamiokande [SK] [5], Chlorine [6], and Gallium [789]), place severe constraints on the neutrino parameters, especially mixing between first and second generations 101112. As an example the neutrino parameter space obtained from the global analysis of all available solar neutrino plus the KamLAND data is shown in Fig. 10 12.

The aim of this short contribution is to reemphasize that, in principle, high-precision solarneutrino data have potential beyond exploring neutrino parameter space. Here we consider two other applications to solar physics and to nuclear physics of the neutrino-target interactions.

Some time ago it was pointed out that solar neutrino data can be inverted to extract information about the density scale height 13 in a similar way the helioseismological information is inverted to obtain the sound-speed profile throughout the Sun. Even though the precision of the data has not yet reached to a point where such an inversion is possible, it is currently possible to obtain rather tight limits on fluctuations of the solar density. To do so one assumes 14 that the electron density $N_{e}$ fluctuates around the value, $\left\langle N_{e}\right\rangle$,

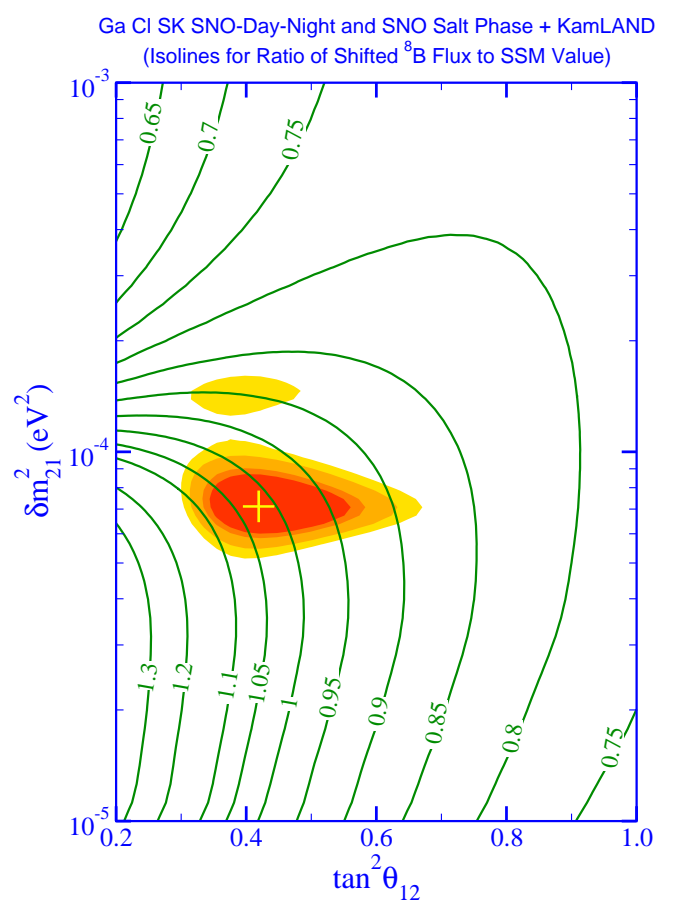

Figure 1. Allowed confidence levels from the joint analysis of all available solar neutrino data (chlorine, average gallium, SNO and SK spectra and SNO salt phase) and KamLAND reactor data The isolines are the ratio of the shifted ${ }^{8} \mathrm{~B}$ flux to the SSM value. At best fit (marked by a cross) the value of this ratio is 1.02 (from Ref. [12]).

*baha@nucth.physics.wisc.edu

†yuksel@nucth.physics.wisc.edu 


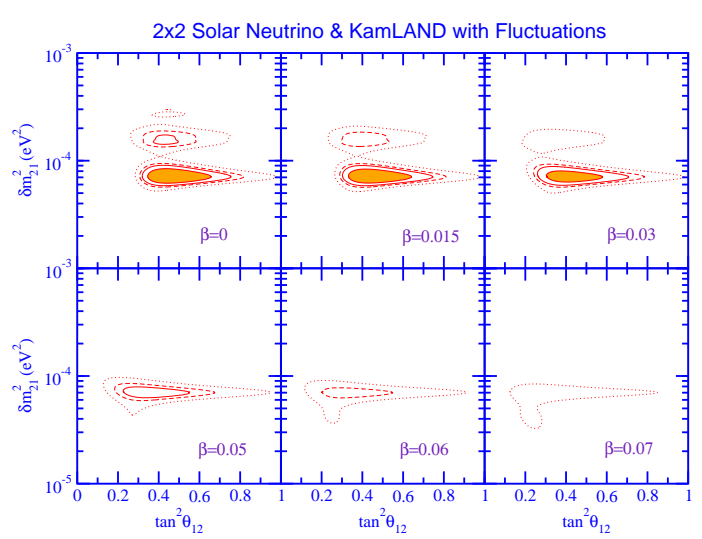

Figure 2. Allowed regions of the neutrino parameter space with solar-density fluctuations when the data from the solar neutrino and KamLAND experiments are used. The SSM density profile of Ref. [15] and the correlation length of $10 \mathrm{~km}$ are used. The case with no fluctuations $(\beta=0)$ are compared with results obtained with the indicated fractional fluctuation. The shaded area is the $70 \%$ confidence level region. $90 \%$ (solid line), $95 \%$ (dashed line), and $99 \%$ (dotted line) confidence levels are also shown (From Ref. [16]).

predicted by the Standard Solar Model (SSM) 15

$N_{e}(r)=(1+\beta F(r))\left\langle N_{e}(r)\right\rangle$,

and that the fluctuation $F(r)$ takes the form of white-noise. The neutrino parameter space for various values of the parameter $\beta$ was calculated in Ref. [16] and is shown in Figure 2. These results, in agreement with the calculations of other authors 1718, show that the neutrino data constrains solar density fluctuations to be less than $\beta=0.05$ at the $70 \%$ confidence level. The best fit to the combined solar neutrino and KamLAND data is given by $\beta=0$ (exact SSM).

In the effective field theory approach to nuclear interactions, nonlocal interactions at short distances are represented by effective local interactions in a derivative expansion. Since the effect of a given operator on low-energy physics is inversely proportional to its dimension, an effective

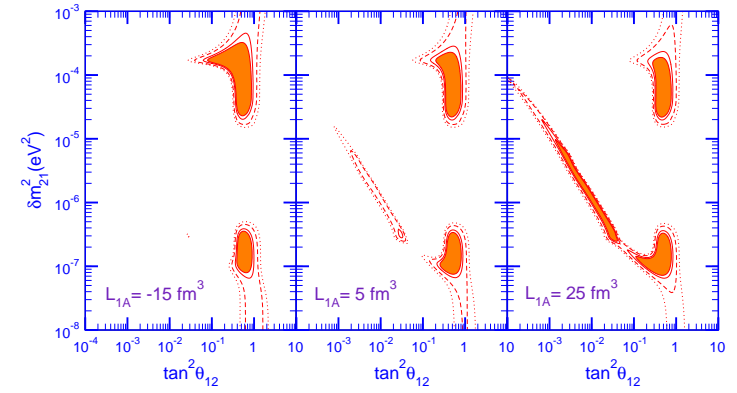

Figure 3. The change in the allowed region of the neutrino parameter space using solar neutrino data measured at SNO as the value of $L_{1 A}$ changes. The shaded areas are the $90 \%$ confidence level region. $95 \%$ (solid line), $99 \%$ (logdashed line), and $99.73 \%$ (dotted-line) confidence levels are also shown (From Ref. 21]).

theory valid at low energies can be written down by retaining operators up to a given dimension. It turns out that one needs to introduce a single coefficient, commonly called $L_{1 A}$, to parameterize the unknown isovector axial two-body current which dominates the uncertainties of all neutrinodeuteron interactions [19. Chen, Heeger, and Robertson, using the Sudbury Neutrino Observatory (SNO) and SuperKamiokande (SK) chargedcurrent, neutral current, and elastic scattering rate data, found [20] $L_{1 A}=4.0 \pm 6.3 \mathrm{fm}^{3}$. In order to obtain this result they wrote the observed rate in terms of an averaged effective cross section and a suitably defined response function. One can explore the phenomenology associated with the variation of $L_{1 A}$. For example the variation of the neutrino parameter space, which fits the SNO data, as $L_{1 A}$ changes was calculated in 21 and is shown in Figure 3. In Ref. 21] the most conservative fit value with fewest assumptions is found to be $L_{1 A}=4.5_{-12}^{+18} \mathrm{fm}^{3}$. It was also shown that the contribution of the uncertainty of $L_{1 A}$ to the analysis and interpretation of the solar neutrino data measured at the Sudbury Neutrino Observatory is significantly less than the uncertainty coming from the lack of having a better knowl- 
edge of $\theta_{13}$, the mixing angle between first and third generations.

In conclusion we would like to reiterate that the utility of the solar neutrino and related reactor and long-baseline neutrino experiments goes well beyond that of exploring neutrino parameter space. In this short note we briefly discussed only two of such applications out of a much longer list.

This work was supported in part by the U.S. National Science Foundation Grant No. PHY0244384 and in part by the University of Wisconsin Research Committee with funds granted by the Wisconsin Alumni Research Foundation.

\section{REFERENCES}

1. S. N. Ahmed et al. [SNO Collaboration], arXiv:nucl-ex/0309004

2. K. Eguchi et al. [KamLAND Collaboration], Phys. Rev. Lett. 90 (2003) 021802 arXiv:hep-ex/0212021.

3. A. Y. Smirnov, arXiv:hep-ph/0311259

4. V. Barger, D. Marfatia and K. Whisnant, Int. J. Mod. Phys. E 12 (2003) 569 arXiv:hep-ph/0308123.

5. S. Fukuda et al. [Super-Kamiokande Collaboration], Phys. Lett. B 539, (2002) 179 arXiv:hep-ex/0205075.

6. B. T. Cleveland et al., Astrophys. J. 496 (1998) 505.

7. J. N. Abdurashitov et al. [SAGE Collaboration], J. Exp. Theor. Phys. 95 (2002) 181 [Zh. Eksp. Teor. Fiz. 122 (2002) 211] arXiv:astro-ph/0204245.

8. W. Hampel et al. [GALLEX Collaboration], Phys. Lett. B 447 (1999) 127.

9. M. Altmann et al. [GNO Collaboration], Phys. Lett. B $\mathbf{4 9 0}$ (2000) 16 arXiv:hep-ex/0006034.

10. A. B. Balantekin and H. Yuksel, J. Phys. G 29 (2003) 665 arXiv:hep-ph/0301072.

11. P. C. de Holanda and A. Y. Smirnov, arXiv:hep-ph/0309299 M. Maltoni, T. Schwetz, M. A. Tortola and J. W. F. Valle, arXiv:hep-ph/0309130 G. L. Fogli, E. Lisi, A. Marrone and A. Palazzo, arXiv:hep-ph/0309100

12. A. B. Balantekin and H. Yuksel,
arXiv:hep-ph/0309079

13. A. B. Balantekin, J. F. Beacom and J. M. Fetter, Phys. Lett. B 427 (1998) 317 arXiv:hep-ph/9712390; see also A. B. Balantekin, Phys. Rept. $315 \quad$ (1999) 123 arXiv:hep-ph/9808281.

14. F. N. Loreti and A. B. Balantekin, Phys. Rev. D 50 (1994) 4762 arXiv:nucl-th/9406003; A. B. Balantekin, J. M. Fetter and F. N. Loreti, Phys. Rev. D 54 (1996) 3941 arXiv:astro-ph/9604061.

15. J. N. Bahcall, M. H. Pinsonneault and S. Basu, Astrophys. J. 555 (2001) 990 arXiv:astro-ph/0010346.

16. A. B. Balantekin and H. Yuksel, Phys. Rev. D 68 (2003) 013006 arXiv:hep-ph/0303169; see also A. B. Balantekin, arXiv:hep-ph/0109163.

17. C. P. Burgess, N. S. Dzhalilov, M. Maltoni, T. I. Rashba, V. B. Semikoz, M. A. Tortola and J. W. F. Valle, arXiv:hep-ph/0310366

18. M. M. Guzzo, P. C. de Holanda and N. Reggiani, Phys. Lett. B 569 (2003) 45 arXiv:hep-ph/0303203.

19. M. Butler and J. W. Chen, Nucl. Phys. A 675 (2000) 575 arXiv:nucl-th/9905059; M. Butler, J. W. Chen and X. Kong, Phys. Rev. C 63 (2001) 035501 arXiv:nucl-th/0008032.

20. J. W. Chen, K. M. Heeger and R. G. H. Robertson, Phys. Rev. C 67 (2003) 025801 arXiv:nucl-th/0210073.

21. A. B. Balantekin and H. Yuksel, Phys. Rev. C 68 (2003) 055801 arXiv:hep-ph/0307227. 